TRANSACTIONS OF THE

AMERICAN MATHEMATICAL SOCIETY

Volume 181, July 1973

\title{
THE LATTICE TRIPLE PACKING OF SPHERES IN EUCLIDEAN SPACE
}

\author{
BY
}

G. B. PURDY

ABSTRACT. We say that a lattice $\Lambda$ in $n$-dimensional Euclidean space $E_{n}$ provides a $k$-fold packing for spheres of radius 1 if, when open spheres of radius 1 are centered at the points of $\Lambda$, no point of space lies in more than $k$ spheres. The multiple packing constant $\Delta_{k}^{(n)}$ is the smallest determinant of any lattice with this property. In the plane, the first three multiple packing constants $\Delta_{2}^{(2)}, \Delta_{3}^{(2)}$, and $\Delta_{4}^{(2)}$ are known, due to the work of Blundon, Few, and Heppes. In $E_{3}, \Delta_{2}^{(3)}$ is known, because of work by Few and Kanagasabapathy, but no other multiple packing constants are known. We show that $\Delta_{3}^{(3)} \leq$ $8 \sqrt{38} / 27$ and give evidence that $\Delta_{3}^{(3)}=8 \sqrt{38} / 27$. We show, in fact, that a lattice with determinant $8 \sqrt{38} / 27$ gives a local minimum of the determinant among lattices providing a 3 -fold packing for the unit sphere in $E_{3}$.

1. Introduction. Let $\Lambda$ be an $n$-dimensional lattice in $n$-dimensional Euclidean space $E_{n}$, such that, if open spheres of radius 1 are centered at the points of $\Lambda$, then no point of space is covered more than $k$ times. That is, for any point $X$ in $E_{n}$ there do not exist distinct points $L_{1}, L_{2}, \ldots, L_{k+1}$ of $\Lambda$ such that $\left|X-L_{1}\right|, \ldots,\left|X-L_{k+1}\right|<1$. Then we say that $\Lambda$ provides a $k$-fold packing for spheres of radius 1 . The terms single, double and triple are synonymous with $k$-fold for $k=1,2$ and 3 .

Let $d(\Lambda)$ denote the determinant of $\Lambda$, and let $\Delta_{k}^{(n)}$ denote the lower bound of $d(\Lambda)$, taken over all lattices $\Lambda$ that provide a $k$-fold packing for spheres of radius 1 . (Thus $\Delta_{1}^{(n)}$ is the critical determinant of a sphere of radius 2.) It is well known and easy to see (e.g., divide one generator of the lattice by $k$ ) that $\Delta_{k}^{(n)} \leq \Delta_{1}^{(n)} / k$.

It has been shown by Few [1] that $\Delta_{2}^{(2)}=(1 / 2) \Delta_{1}^{(2)}$, and Heppes [5] showed that $\Delta_{k}^{(2)}=\Delta_{1}^{(2)} / k$ if and only if $k \leq 4$.

In [4] Few and Kanagasabapathy determined the exact value of $\Delta_{2}^{(3)}$, namely $3 \sqrt{3} / 2$, which is less than $\Delta_{1}^{(3)} / 2=2 \sqrt{2}$. By constructing particular lattices they also showed that $\Delta_{2}^{(n)}<\Delta_{1}^{(n)} / 2$ for every $n \geq 3$.

Few remarks in [2] that $\Delta_{2}^{(3)}$ is the only multiple packing constant known exactly in three dimensions or more, and in this note I shall prove that $\Delta_{3}^{(3)} \leq$

Received by the editors September 29, 1972 .

AMS (MOS) subject classifications (1970). Primary 10E 30.

Key words and phrases. Spheres, lattice packing, multiple packing. 
$8 \sqrt{38} / 27<\Delta_{1}^{(3)} / 3=4 \sqrt{2} / 3$ and give evidence suggesting that $\Delta_{3}^{(3)}=8 \sqrt{38} / 27$. In fact, I prove

Theorem 1. A certain lattice $\Lambda_{0}$ of determinant $d_{0}=8 \sqrt{38} / 27$ provides a triple packing for the unit spbere $S$. Also $\Lambda_{0}$ bas generators $P, Q, R$ with $|P|=2 / 3$.

Theorem 2. Any lattice $\Lambda$ baving generators $P^{\prime}, Q^{\prime}, R^{\prime}$ with $\left|P^{\prime}\right| \leq 0.95$ providing a triple packing for $S$ must bave determinant $d(\Lambda) \geq d_{0}$ with equality only when $\Lambda=\Lambda_{0}$. Hence $\Lambda_{0}$ gives a local minimum of $d(\Lambda)$ for triple packing of unit spheres.

Remark. There is extensive numerical evidence that $d(\Lambda)$ does not fall below $d_{0}$ for any triple packing with $S$.

\section{An economical lattice $\Lambda_{0}$.}

Theorem 1. The best lattice triple packing for spberes in $E^{3}$ bas determinant $d(\Lambda) \leq 8 \sqrt{38} / 27=\sqrt{2432 / 729}=1.82649 \cdots$, since indeed the lattice $\Lambda_{0}$ generated by $P, Q$ and $R$ where $P=(a, 0,0)=(2 / 3,0,0), Q=(b, b, 0)=(1 / 3, \sqrt{3}, 0)$ and $R=(g, f, c)$, where $g=1 / 3, f=(11 \sqrt{3}) / 27$ and $c^{2}=3-f^{2}$, provides a triple packing for the unit sphere $S$.

Proof. Convention: The letters $\lambda, \mu$ and $\nu$ will denote integers. $S(A, r)$ will be the open sphere of radius $r$ centered at $A ; S(A)$ will denote $S(A, 1)$; thus $S=S$ (origin). Suppose that the point $X=(x, y, z)$ is covered four times. Translating $X$ by a lattice point, we may suppose that $X \in S$, and replacing $X$ by $-X$ if necessary we may suppose $z \geq 0$. The three other spheres covering $X$ can be written $S(\lambda P+\mu Q+\nu R)=S+\lambda P+\mu Q+\nu R$ where $(\lambda, \mu, \nu) \neq(0,0,0)$. We must have $|\lambda P+\mu Q+\nu R|<2$ since they must intersect $S$. Therefore

$$
(\lambda a+\mu b+\nu g)+(\mu b+\nu f)^{2}+\nu^{2} c^{2}<4
$$

and $|\nu|<2 / c$. Since $c>1$, we have $\nu \in\{-1,0,1\}$. Now $\nu$ cannot be -1 , since otherwise $|X-(\lambda P+\mu Q-R)| \geq|c+z|>1$. Hence $\nu \in\{0,1\}$. From (*) we also get $|\mu b+\nu f|<2$. Since $0 \leq \nu \leq 1$ and $0 \leq f \leq b / 2$ and $b=\sqrt{3}>4 / 3$ this gives $-2<\mu<2, \mu \in\{-1,0,1\}$. We divide the proof into two parts.

Part 1. $y \geq 0$. Then $\mu \in\{0,1\}$; in fact if $\mu=-1$, then for $X \in S(\lambda P+\mu Q+\nu R)$ we would have $|X-(\lambda P-Q+\nu R)|^{2} \geq(b+y-\nu f)^{2}>(16 b / 27)^{2}=256 / 243$, since $\nu \in\{0,1\}$. Also $(\mu, \nu) \neq(1,1)$, since $|\lambda P+Q+R|^{2} \geq b^{2}+c^{2}>4$. Hence $(\mu, \nu) \in\{(0,0),(1,0),(0,1)\}$.

Type 1 spheres. Suppose $(\mu, \nu)=(0,0)$. Then $\lambda P+\mu Q+\nu R=\lambda P$, and $S(\lambda P) \cap S=\varnothing$ if $|\lambda|>2$, since $|3 P|=3 a=2$. The $S(\lambda P)$ such that $0<|\lambda| \leq 2$ are called Type 1 spheres. 
Type 2 spheres. Suppose $(\mu, \nu)=(1,0)$. Then $\lambda P+\mu Q+\nu R=\lambda P+Q$, and $S(\lambda P+Q) \cap S=\varnothing$ if $\lambda \notin\{0,-1\}$, since then $|\lambda P+Q|^{2}=b^{2}+(\lambda a+b)^{2}=3+$ $|2 \lambda / 3+1 / 3|^{2} \geq 4$. The $S(Q-P)$ and $S(Q)$ are called Type 2 spheres.

Type 3 spheres. Suppose $(\mu, \nu)=(0,1)$. Then $\lambda P+\mu Q+\nu R=R+\lambda P$, $S(R+\lambda P) \cap S=\varnothing$ if $\lambda \notin\{0,-1\}$. To see this, observe that if $S(R+\lambda P) \cap S \neq \varnothing$ then $(\lambda a+g)^{2}+f^{2}+c^{2}<4$; since $f^{2}+c^{2}=3,|2 \lambda / 3+1 / 3|<1$ and $\lambda \in\{0,-1\}$. We call $S(R)$ and $S(R-P)$ Type 3 spheres.

It follows from the discussion of Type 2 and Type 3 spheres that $S \cap S(\lambda P+E)=\varnothing$ if $\lambda \notin\{-1,0\}$ where $E \notin\{R, Q\}$. In particular,

$$
\begin{aligned}
& \varnothing=S \cap S(E+P)=S(-P) \cap S(E)=S(-2 P) \cap S(E-P), \\
& \varnothing=S \cap S(E+2 P)=S(-2 P) \cap S(E), \\
& \varnothing=S \cap S(E-2 P)=S(P) \cap S(E-P)=S(2 P) \cap S(E), \\
& \varnothing=S \cap S(E-3 P)=S(2 P) \cap S(E-P) .
\end{aligned}
$$

From the discussion of Type 1 spheres $S \cap S(\lambda P)=\varnothing$ for $|\lambda|>2$ so that

$$
\begin{aligned}
& \varnothing=S \cap S(3 P)=S(-P) \cap S(2 P)=S(-2 P) \cap S(P), \\
& \varnothing=S \cap S(4 P)=S(-2 P) \cap S(2 P) .
\end{aligned}
$$

We now draw a graph $G$ where edges $A$ and $B$ are joined only if we know that $S(A) \cap S(B)=\varnothing$.

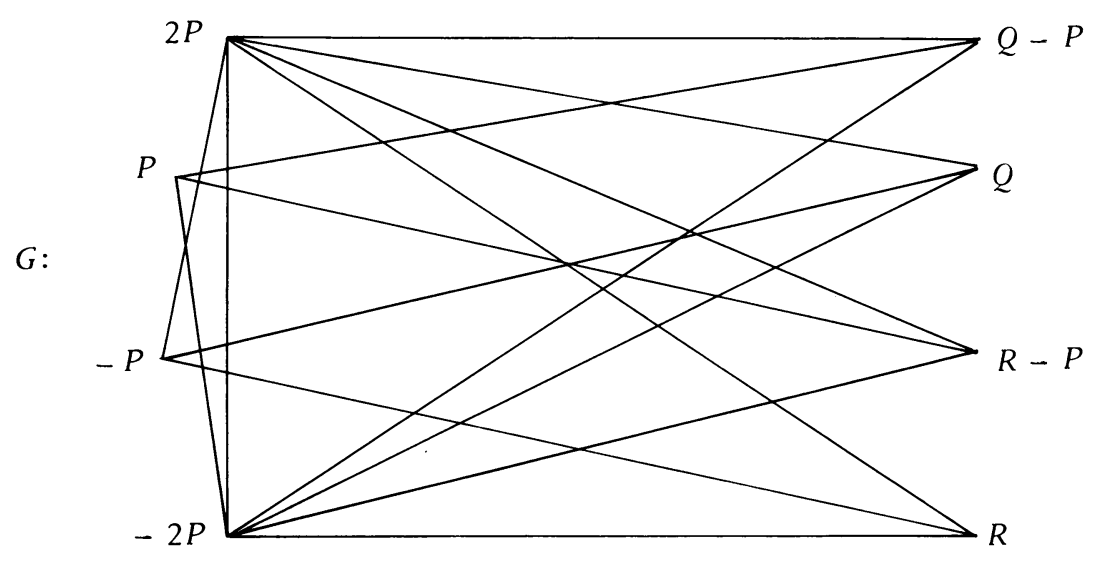

We next observe that $\varnothing=S(Q+\lambda P) \cap S \cap S\left(R+\lambda^{\prime} P+\nu Q\right)$. For $|Q+\lambda P| \geq|Q|=$ $\sqrt{b^{2}+b^{2}}>\sqrt{3}$. Therefore the height (maximal value of the $z$ coordinate of the closure) of $S(Q+\lambda P) \cap S$ is less than $\sqrt{1-3 / 4}=1 / 2<c-1$, since $c=1.5 \cdots>3 / 2$. Since $R+\lambda^{\prime} P$ has $z$ component $c$, the above intersection is void. Hence we cannot have $X$ simultaneously inside a sphere of Type 2 and a sphere of Type 3, so 
$X \in S\left(\lambda_{1} P\right) \cap S\left(\lambda_{2} P\right) \cap S\left(E+\lambda_{3} P\right) \quad$ with $0<\left|\lambda_{1}\right|, \quad\left|\lambda_{2}\right| \leq \bar{z},-1 \leq \lambda_{3} \leq 0$, or

$X \in S\left(\lambda_{1} P\right) \cap S\left(E+\lambda_{2} P\right) \cap S\left(E+\lambda_{3} P\right) \quad$ with $0<\left|\lambda_{1}\right| \leq 2,-1 \leq \lambda_{2}, \lambda_{3} \leq 0$, where $E \in\{R, Q\}$. Both of these contradict the graph $G$, and Part 1 follows.

Part 2. We now suppose that $y<0$. Recall that if $S \cap S(\lambda P+\mu Q+\nu R) \neq \varnothing$, then $-1 \leq \mu \leq 1$ and $0 \leq \nu \leq 1$. For those $S(\lambda P+\mu Q+\nu R)$ containing $X$ we must have $-1 \leq \mu \leq 0$. For suppose that $\mu=1$; since $X=(x, y, z), y<0$, we would have $|\lambda P+\mu Q+\nu R-X| \geq|b+\nu f-y|>b>1$. The spheres $S(\lambda P+\mu Q+\nu R)$ containing $X$ other than $S$ may therefore be divided into four types, as follows:

Type 1 spheres, when $(\mu, \nu)=(0,0)$. As before the only spheres $S(\lambda P)$ intersecting $S$ satisfy $0<|\lambda| \leq 2$, i.e., the Type 1 spheres are $S(2 P), S(P), S(-P)$ and $S(-2 P)$.

Type 2 spheres, when $(\mu, \nu)=(-1,0)$. If $S(\lambda P+\mu Q+\nu R)$ is to intersect $S$ we must have $4>|\lambda P+\mu Q+\nu R|^{2}=(\lambda a-b)^{2}+b^{2}=(2 \lambda / 3-1 / 3)^{2}+3$. Hence $0 \leq \lambda \leq 1$, i.e., the Type 2 spheres are $S(-Q)$ and $S(P-Q)$.

Type 3 spheres, when $(\mu, \nu)=(0,1)$. As in Part $1,-1 \leq \lambda \leq 0$ if $S(\lambda P+\mu Q+\nu R)$ intersects $S$, i.e., the Type 3 spheres are $S(R)$ and $S(R-P)$.

Type 4 spheres, when $(\mu, \nu)=(-1,1)$. If $S(\lambda P+\mu Q+\nu R)$ intersects $S$, then $(\lambda a+g-b)^{2}+(f-b)^{2}+c^{2}<4,4 \lambda^{2} / 9<4-(b-f)^{2}-c^{2}=4 / 9, \lambda^{2}<1, \lambda=0$, and $S(R-Q)$ is the only sphere of Type 4 .

As we did in Part 1, we deduce several new disjoint pairs of spheres. From the discussion of Type 4 spheres, $S \cap S(-Q+R+\lambda P)=\varnothing$ if $\lambda \neq 0$, so we have $S(\lambda P) \cap S(-Q ; R)=\varnothing$ if $\lambda \neq 0$. From the Type 2 spheres we have

$$
\begin{aligned}
& \varnothing=S \cap S(-Q+2 P)=S(-P) \cap S(-Q+P)=S(-2 P) \cap S(-Q), \\
& \varnothing=S \cap S(-Q+3 P)=S(-2 P) \cap S(-Q+P), \\
& \varnothing=S \cap S(-Q-P)=S(P) \cap S(-Q)=S(2 P) \cap S(-Q+P), \\
& \varnothing=S \cap S(-Q-2 P)=S(2 P) \cap S(-Q) .
\end{aligned}
$$

If we combine these with some of the disjoint pairs that we already know from Part 1 and draw a graph, $G^{\prime}$, in which $A$ is joined to $B$ only if we know $S(A) \cap S(B)=\varnothing$, we obtain the following graph.

In addition to these disjoint spheres, we observe that, for any $\lambda$ and $\lambda^{\prime}$, $S(-Q+\lambda P) \cap S\left(R+\lambda^{\prime} P\right) \cap S=\varnothing$, since $|-Q+\lambda P| \geq|Q|$, so that the height of $S(-Q+P) \cap S$ is not greater than the height of $S \cap S(Q)$, which is less than $1 / 2<c-1$, and $c$ is the height of $R+\lambda^{\prime} P$.

Also, for any $\lambda, \varnothing=S \cap S(Q+\lambda P) \cap S(R)=S \cap S(-Q-\lambda P) \cap S(-R)=$ $S(R) \cap S(R-Q-\lambda P) \cap S$.

In particular,

$$
S(R) \cap S(R-Q) \cap S=\varnothing .
$$




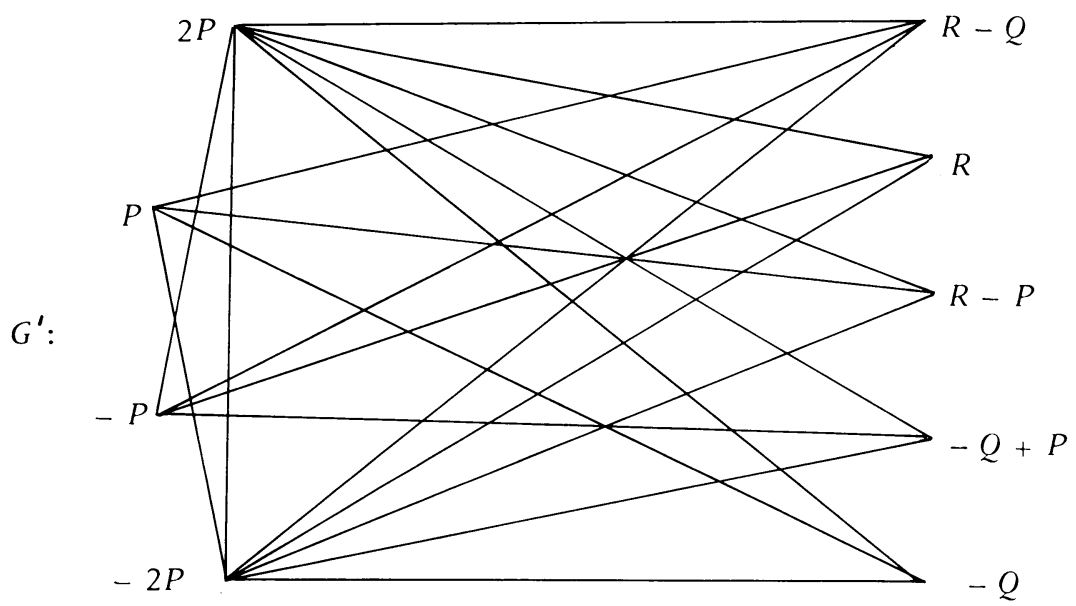

The following enumeration of possibilities shows that $X$ cannot be contained in the necessary spheres, and Theorem 1 follows:

Clearly Types 2 and 3 cannot both occur by the fourth paragraph above, and two spheres of Type 1 cannot occur with anything else by the graph $G^{\prime}$. Again by the graph $G^{\prime}$, if two spheres of Type 2 (or two spheres of Type 3) occur, the remaining sphere cannot have Type 1 . Hence the only remaining possibilities are

$$
\begin{gathered}
X \in S\left(\lambda_{1} P\right) \cap S\left(\lambda_{2} P-Q\right) \cap S(R-Q), \quad 0<\left|\lambda_{1}\right| \leq 2,0 \leq \lambda_{2} \leq 1, \\
X \in S\left(\lambda_{1} P\right) \cap S\left(\lambda_{2} P+R\right) \cap S(R-Q), \quad 0<\left|\lambda_{1}\right| \leq 2,-1 \leq \lambda_{2} \leq 0, \\
X \in S(P-Q) \cap S(-Q) \cap S(R-Q), \quad \text { or } \\
X \in S(R) \cap S(-P+R) \cap S(R-Q) .
\end{gathered}
$$

Now (2) and (3) contradict $G^{\prime}$, and (1) excludes (5). To eliminate (4) we observe that, since $S(P) \cap S \cap S(R) \cap S(Q)=\varnothing$, we must have $S(P-Q) \cap S(-Q) \cap S(R-Q) \cap S=\varnothing$.

3. The lattice $\Lambda_{0}$ is locally optimal.

Remark 1. An arbitrary lattice $\Lambda$ in $E_{3}$ has a basis $P, Q, R$ where $|P| \leq$ $|Q| \leq|R|$ are the successive minima of the unit sphere, $P=(a, 0,0), Q=(b, b, 0)$, $R=(g, f, c), a, b, c>0,0 \leq b \leq a / 2,0 \leq f \leq b / 2$, and $-a / 2<g \leq a / 2$. Such a basis is said to be reduced in the sense of Gauss or simply reduced. For a proof, see [6, p. 163 et seq., "Seebers inequality"].

Remark 2. If $\Lambda$ has a reduced basis $P, Q, R$ with $P=(a, 0,0), Q=(b, b, 0)$, $R=(g, f, c)$ and if $d(\Lambda) \leq d_{0}$, then $b^{2} \leq b_{m}^{2}$, where $b_{m}^{2}=a^{2} / 6+(2 / 3) \sqrt{a^{4} / 16+3 d_{0}^{2} / a^{2}}$.

Proof. Using $\left|R^{2}\right|=g^{2}+f^{2}+c^{2} \geq|Q|^{2}=b^{2}+b^{2}$, and the other inequalities of reduction, we have $d_{0}^{2} \geq d^{2}(\Lambda)=a^{2} b^{2} c^{2} \geq a^{2} b^{2}\left(b^{2}+b^{2}-g^{2}-f^{2}\right) \geq$ 
$a^{2} b^{2}\left(3 b^{2} / 4-a^{2} / 4\right)$. Putting $t=b^{2}$, we get $3 a^{2} t^{2}-a^{4} t-4 d_{0}^{2} \leq 0$. Hence $b^{2}$ must lie between the roots $a^{2} / 6-(2 / 3) \sqrt{a^{4} / 16+3 d_{0}^{2} / a^{2}}$ and $a^{2} / 6+(2 / 3) \sqrt{a^{4} / 16+3 d_{0}^{2} / a^{2}}$ of the quadratic.

Let $\rho^{+}$denote $\max \{0, \rho\}$.

Theorem 2. If $(\Lambda, S)$ is a triple packing and $P=(a, 0,0), Q=(b, b, 0)$ and $R=(g, f, c)$ gives a basis for $\Lambda$ reduced in the sense of Gauss, and $a \leq 1$, then

(6) $d(\Lambda)=a b c \geq a b \sqrt{\left\{4-(a+b)^{2}-\left(\left(b^{2}-2 a b-b^{2}\right) /(2 b)\right)^{2}\right\}^{+}}=f_{1}(a, b, b)$

when $0 \leq g \leq h$, and

$$
\left.d(\Lambda)=a b c \geq a b \sqrt{\left\{4-9 a^{2} / 4-\left(b^{2}+b^{2}-3 a b\right)^{2} /(2 b)^{2}\right.}\right\}^{+}=f_{2}(a, b, b)
$$

when $-a / 2 \leq g \leq 0$ and when $b \leq g \leq a / 2$. Furthermore $f_{1}(a, b, b) \geq f_{2}(a, b, b)$, so that in fact

$$
d(\Lambda) \geq f_{2}(a, b, b)
$$

in all cases. Also, if $d(\Lambda) \leq d_{0}$ and $2 / 3 \leq a \leq 0.9508$, we have

$$
f_{2}^{2}(a, b, b) \geq \min \left\{p(a), d_{0}^{2}+1 / 100\right\},
$$

where $p(a)=2 a^{6}-11 a^{4}+12 a^{2}, p(2 ; 3)=d_{0}^{2}$, and

$$
p(a)>d_{0}^{2} \text { for } 2 / 3<a \leq 0.9508 \text {. }
$$

Hence $d(\Lambda) \geq d_{0}$ for $2 / 3 \leq a \leq 0.9508$, and with equality only if $a=2 / 3$.

Proof. Suppose that $(\Lambda, S)$ gives a triple packing and that $P, Q, R$ form a reduced basis of $\Lambda$. From reduction, we have

$$
|P| \leq|Q| \leq|R|, 0 \leq b \leq a / 2,0 \leq f \leq b / 2 \text {, and }|g| \leq a / 2 \text {. }
$$

We also have $a \geq 2 / 3$, since otherwise the point $(1 / 2) P$ would be covered by $S(-P), S, S(P)$ and $S(2 P)$. Observe that the center of the parallelogram with vertices $P, Q, Q+P$ and the origin will be covered by the four spheres $S, S(P)$, $S(Q)$, and $S(Q+P)$ unless one of the diagonals $|Q+P|,|Q-P|$ is at least 2 . Since $|Q+P| \geq|Q-P|$ by (11), it follows that $4 \leq|Q+P|^{2}=b^{2}+(a+b)^{2}$; hence

$$
b^{2} \geq 4-(a+b)^{2} \text { and } a \geq 2 / 3
$$

Case 1. In this case we assume

(13) $0 \leq g \leq h$.

A consequence of (13) is that $|R-Q-P|$ is not less than $|R-Q+P|$. They 
cannot both be less than 2 , since then the center of the parallelogram having vertices $R, Q, Q+P, R+P$ would be covered four times. Hence we have

(14) $|R-Q-P| \geq 2$.

Another consequence of (13) is that $|R+P|$ is not less than $|R-P|$. Considering the parallelogram with vertices $P, R, R+P$ and the origin shows that

(15) $|R+P| \geq 2$.

With a view to proving (6) we imagine $a, b, b$ to be fixed and find the point $R=(g, f, c)$ having least nonnegative $c$ such that (13), (14) and (15) hold and also (16) $0 \leq f \leq b / 2$.

We are in fact looking for the lowest point $X=(x, y, z)$ inside the rectangular prism given by

(17) $Q \leq x \leq b, 0 \leq y \leq b / 2, z \geq 0$, subject to the additional constraint

(18) $|X-P-Q| \geq 2,|X+P| \geq 2$.

The problem is somewhat simplified by the fact that the centers $P+Q,-P$ of the spheres lies on the plane $z=0$, outside the prism.

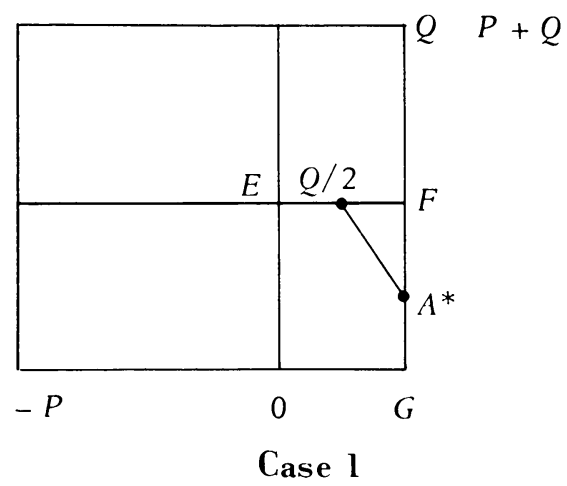

If the right-hand side of $(6)$ is zero, there is nothing to prove. Let us suppose, therefore, that it is positive. We shall show that the point $X^{*}$ that lies on the intersection of the boundary of $S(-P, 2)$ and $S(P+Q, 2)$ and the plane $x=b$ is the lowest point satisfying (17) and (18). We start by finding $X^{*}$. Let $X^{*}=$ $\left(x^{*}, y^{*}, z^{*}\right)$. Let $\pi$ be the radical plane of $S(-P, 2)$ and $S(P+Q, 2)$ (the plane obtained by subtracting the equations of the two spheres). Then $\pi$ passes through $(1 / 2) Q=(b / 2, b / 2,0)$, which is halfway between the center of the two spheres, and has the equation $y-b / 2=(-(2 a+b) / b)(x-b / 2)$. Putting $x^{*}=b$, we obtain $y^{*}=$ $b / 2-\left(2 a b+b^{2}\right) /(2 b)$. We must show that $0 \leq y^{*}$ so that (17) is satisfied. By (12) we have $b^{2} \geq 4-(a+b)^{2} \geq 2 a b+b^{2}+1 / 2$, since $b \leq a ; 2$; hence $2 b y^{*}=b^{2}-\left(2 a b+b^{2}\right)>0$ and (17) follows. We see that $z^{*}=\sqrt{4-(a+b)^{2}-\left(\left(b^{2}-2 a b-b^{2}\right) /(2 b)\right)^{2}}>0$ by a previous assumption. 
The first step in showing that $X^{*}$ is optimal is to show that the bottom of the prism is covered by $S(-P, 2)$ and $S(P+Q, 2)$. This means that there is no $X$ satisfying (17) and (18) with $z=0$. Let $A^{*}=\left(x^{*}, y^{*}, 0\right), E=(0, b / 2,0)$, $F=(b, b / 2,0)$ and $G=(b, 0,0)$. Then $2=\left|P+Q-X^{*}\right|>\left|P+Q-A^{*}\right| \geq|P+Q-F|$, and also $|P+Q-(1 / 2) Q|<2$, since the spheres $S(-P, 2)$ and $S(P+Q, 2)$ intersect and $(1 / 2) Q$ is halfway between their centers. Hence the triangle with vertices $(1 / 2) Q$, $A^{*}, F$ lies in the interior of $S(P+Q, 2)$.

Similarly, $2=\left|-P-X^{*}\right|>\left|-P-A^{*}\right| \geq|-P-G| \geq|-P|$ and $2>|-P-(1 / 2) Q| \geq|-P-E|$ so that the convex pentagon with vertices $G, A^{*},(1 / 2) Q, E$ and the origin lies in the interior of $S(-P, 2)$. Hence the bottom of the prism is covered.

We now let $X_{1}=\left(x_{1}, y_{1}, z_{1}\right)$ be a lowest point satisfying (17) and (18). We know that $X_{1}$ exists, because the set of solutions is nonempty and closed. The point $X_{1}$ must be on the boundary of $S(-P, 2)$ or $S(P+Q, 2)$ since otherwise it could be lowered and still satisfy (18).

Let us suppose first that $X_{1}$ is on the boundary of $S(-P, 2)$. We shall deduce that $X_{1}$ is on the boundary of $S(P+Q, 2)$. Suppose not. Then $\left(x_{1}, y_{1}\right)$ must be the point satisfying (17) that is farthest from $-P$, namely $(b, b / 2)$. But then the point $X_{1}=\left(b, b / 2, \sqrt{4-(b+a)^{2}-b^{2} / 4}\right)$ is easily seen to be inside $S(P+Q, 2)$, contrary to (18).

Suppose that $X_{1}$ is not on the boundary of $S(-P, 2)$. Then $\left|X_{1}-P-Q\right|=2$, and $X_{1}=\left(0,0, \sqrt{4-(a+b)^{2}-b^{2}}\right)$ lies inside $S(-P, 2)$ contrary to (18).

Hence $X_{1}$ lies on the arc of the intersection of $S(-P, 2)$ and $S(P+Q, 2)$ with $z \geq 0$. The highest point of the arc is the point directly above $(1 / 2) Q$, which is on the boundary of the prism, and the lowest point in the prism is $X^{*}$, where the arc cuts the $x=b$ plane. Hence $X_{1}=X^{*}$ and (6) follows.

Case 2. Assume

(19) $-a / 2 \leq g \leq 0$.

Since the center of the parallelogram with vertices $R, Q-P, R+P, Q$ must not be covered four times, we know that one of its two diagonals $|R-Q|,|R-Q+2 P|$ must be at least 2. Assuming Gauss reduction, we always have $|R-Q+2 P| \geq$ $|R-Q|$, since the vectors $R-Q+2 P$ and $R-Q$ differ only in the first component, and $|g-b+2 a| \geq 2 a-|g|-b \geq a \geq|g-b|$. Hence $|R-Q+2 P| \geq 2$.

As in Case 1, one of $|R+P|,|R-P|$ must be at least 2, and from (19) we know that $|R-P| \geq|R+P|$ so that $|R-P| \geq 2$.

In a manner similar to Case 1 , we are looking for the lowest point $X=(x, y, z)$ inside the rectangular prism given by

such that

(20) $-a / 2 \leq x \leq 0,0 \leq y \leq b / 2, z \geq 0$,

(21) $|X-P| \geq 2$, and $|X-Q+2 P| \geq 2$. 


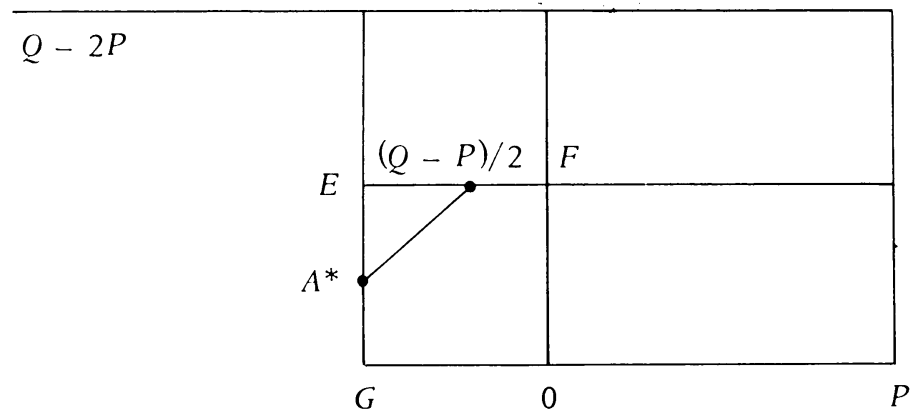

Case 2

The procedure is the same as in Case 1. We may suppose that the right-hand side of (7) is positive. Let $X^{*}=\left(x^{*}, y^{*}, z^{*}\right)$ be the point on the two spheres and the plane $x=-a / 2$, with $z^{*} \geq 0$.

The radical plane $\pi$ of the two spheres passes through $(1 / 2)(Q-P)$. The equation of $\pi$ is $2(b-3 a) x+2 b y=b^{2}+(b-3 a)(b-a)$. Putting $x=x^{*}=-a^{\prime} 2$ yields $y^{*}=\left(b^{2}+b^{2}-3 a b\right) /(2 b)$. We must show that $0 \leq y \leq b / 2$. Now $b^{2} \geq 4-(a+b)^{2}$ for a triple packing; hence $2 b y^{*}=b^{2}+b^{2}-3 a b \geq 4-a^{2}-5 a b \geq 1 / 2$. On the other hand $b^{2} \leq a b / 2 \leq 3 a b, 2 b y^{*}=b^{2}+b^{2}-3 a b \leq b^{2}, y^{*} \leq b / 2$. We see that $z^{*}=$ $\sqrt{4-(3 a / 2)^{2}-\left(\left(b^{2}+b^{2}-3 a b\right) /(2 b)\right)^{2}}>0$ by assumption.

We now show that the bottom of the prism is covered by the two spheres $S(P, 2)$ and $S(Q-2 P, 2)$. Let $A^{*}=\left(x^{*}, y^{*}, 0\right), E=(-a / 2, b / 2,0), F=(0, b / 2,0)$ and $G=(-a / 2,0,0)$. Then $2=|Q-2 P-A|>\left|Q-2 P-A^{*}\right| \geq|Q-2 P-E|$, and also $|(Q-2 P)-(1 / 2)(Q-P)|<2$ since the spheres $S(P, 2)$ and $S(Q-P, 2)$ intersect and $(1 / 2)(Q-P)$ is halfway between their centers, Hence the triangle with vertices $(1 / 2)(Q-P), E, A^{*}$ lies in the interior of $S(Q-2 P, 2)$. Similarly $2>|P-(1 / 2)(Q-P)| \geq|P-F| \geq|P|$ and $2>\left|P-A^{*}\right| \geq|P-G|$, so that the convex pentagon with vertices $G, A^{*},(1 / 2)(Q-P), F$, and the origin lies in the interior of $S(P, 2)$. Hence the bottom of the prism is covered.

We now let $X_{1}=\left(x_{1}, y_{1}, z_{1}\right)$ be a lowest point satisfying (20) and (21); $x_{1}$ exists because the set of points satisfying (20) and (21) is closed and nonempty. Since $z_{1}>0, X_{1}$ must be on the boundary of one of the spheres. We suppose first that $\left|X_{1}-P\right|=2$, and $\left|X_{1}-Q+2 P\right|>2$. Then $\left(x_{1}, y_{1}\right)$ must be as far from $(a, 0)$ as possible still satisfying $(20)$. Hence $X_{1}=\left(-a / 2, b / 2, \sqrt{4-b^{2} / 4-(3 a / 2)^{2}}\right)$, and calculation shows that $\left|X_{1}-Q+2 P\right|<2$, which is a contradiction.

Suppose now that $\left|X_{1}-P\right|>2$, so that $\left|X_{1}-Q+2 P\right|=2$. Then $\left(x_{1}, y_{1}\right)$ must be as far as possible from $(b-2 a, b)$ and still satisfy (20). Now $-2 a \leq$ $b-2 a \leq-(3 / 2) a<-a / 2$. Hence $X_{1}=\left(0,0, \sqrt{4-(b-2 a)^{2}-b^{2}}\right)$. Hence $\left|X_{1}-P\right|^{2}=a^{2}+4-(b-2 a)^{2}-b^{2}<a^{2}+4-9 a^{2} / 4<4$, which is a contradiction. 
Hence $X_{1}$ must be on the boundary of both spheres. In a manner similar to Case $1, X_{1}$ lies on a circular arc whose highest point $(1 / 2)(Q-P)$ is on the boundary of the prism and whose lowest point inside the prism is $X^{*}$. Hence $X_{1}=X^{*}$ and (7) is proved for this case.

Case 3. Assume

(22) $b \leq g \leq a / 2$.

The vectors $R-Q+P, R-Q-P$ differ only in the first component and, by (22), $|g-b+a| \geq|g-b-a|$. Since the center of the parallelogram with vertices $P, R-Q, R-Q+P$ and the origin must not be covered four times, we conclude that $|R-Q+P| \geq 2$. On the other hand, as in Case $1,|R+P| \geq 2$.

We are seeking the lowest point $X_{1}$ of the prism

(23) $b \leq x \leq a / 2,0 \leq y \leq b / 2, z \geq 0$,

such that

(24) $|X+P| \geq 2,|X-Q+P| \geq 2$.

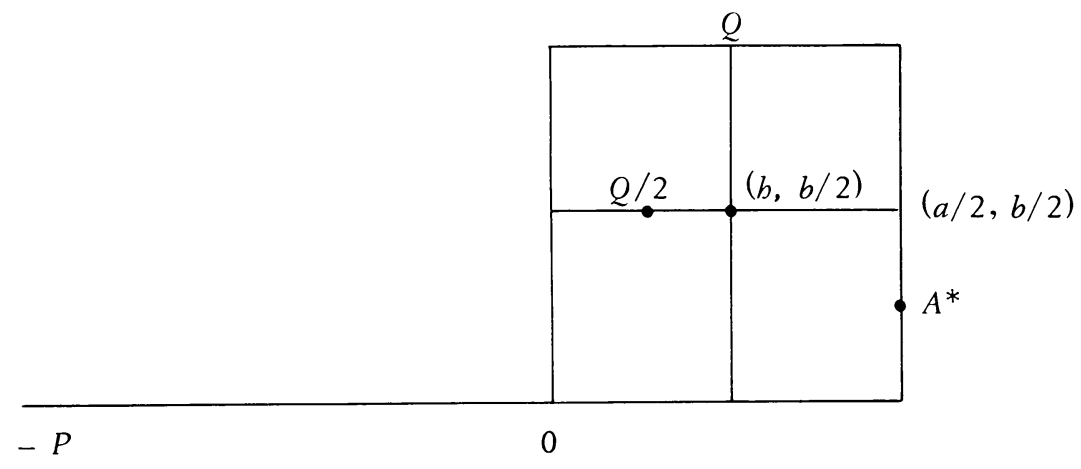

Case 3

If the right-hand side of (7) is zero, there is nothing to prove. Let us suppose that it is positive. Let $X$ be the point on the two spheres and the plane $x=a / 2$. To find $X=(x, y, z)$ we solve $9 a^{2} / 4+y^{2}+z^{2}=4,(3 a / 2-b)^{2}+(y-b)^{2}+z^{2}=4$, and obtain $x^{*}=a / 2, y^{*}=\left(b^{2}+b^{2}-3 a b\right) /(2 b)$, and $z^{*}=\sqrt{4-9 a^{2} / 4-\left(y^{*}\right)^{2}}$. This is the same $y^{*}$ that appears in Case 2 , so $0 \leq y^{*} \leq b / 2$ and $X^{*}$ satisfies (23) and (24).

Now let $X_{1}$ be a lowest point satisfying (23) and (24), and we shall show that $X_{1}=X^{*}$.

We must show that the bottom of the prism is covered. Since $x=a / 2$ maximizes the horizontal distance from both $-P$ and $Q-P$, it is sufficient to show that the line segment $\{(a / 2, t, 0): 0 \leq t \leq b / 2\}$ is covered. The spheres $S(-P, 2)$ and $S(Q-P, 2)$ intersect the plane $x=a / 2$ in circles which intersect at $\left(a^{\prime} 2, y^{*}, z^{*}\right)$. Since $z^{*}>0$, the segment is covered.

For a lowest point $X_{1}$ we must have $x_{1}=a / 2$. Since the spheres intersect 
the $x=a / 2$ plane in circles whose centers have $y$ components 0 and $b$ respectively, it is clear that $X_{1}=X^{*}$ and inequality (7) holds. This finishes Case 3.

Hence $d(\Lambda) \geq \min \left\{f_{1}, f_{2}\right\}$. We observe immediately that $f_{1} \geq f_{2}$. This would follow if $\psi(b) \geq 0$ where $\psi(b)=9 a^{2} / 4+(b / 2-(3 a-b) b /(2 b))^{2}-(b+a)^{2}-\{(b / 2-(b / 2)(b+2 a) / b)\}^{2}$. Now $\psi(0)=9 a^{2} / 4-a^{2}=(5 / 4) a^{2}>0$ and $\psi(a / 2)=0$. Differentiating, we have $\psi^{\prime}(b)=-\left\{(5 a) /\left(2 b^{2}\right)\right\}\left(b^{2}+3 b^{2}-a b\right)<0$ since $b^{2} \geq a^{2}-b^{2} \geq(3 / 4) a^{2}$.

We now prove

$$
f_{2}^{2} \geq \min \left\{d_{0}^{2}+1 / 100,2 a^{6}-11 a^{4}+12 a^{2}\right\}
$$

under the hypothesis that $d(\Lambda) \leq d_{0}$ and $2 / 3 \leq a \leq 0.9508$. Write $F(a, b, b)=f_{2}^{2}=$ $a^{2} b^{2}\left\{4-9 a^{2} / 4-(b / 2+b(b-3 a) /(2 b))^{2}\right\}$ and put $t=b^{2}$. Clearly $\partial^{2} F / \partial t^{2}=$ $-a^{2} / 2<0$. Hence $F(a, b, b) \geq \min \{\phi(a, b), \psi(a, b)\}$, where $\phi(a, b)=F\left(a, b, \sqrt{4-(a+b)^{2}}\right)$, and $\psi(a, b)=F\left(a, b, b_{m}\right)$, where $b_{m}$ was defined in Remark 2. Now

$$
\phi(a, b)=a^{2}\left\{4-(a+b)^{2}\right\}\left\{4-9 a^{2} / 4-(1 / 4)\left(4-(a+b)^{2}\right)-(1 / 2) b(b-3 a)\right\}-\left(a^{2} / 4\right) b^{2}(b-3 a)^{2}
$$

and calculation shows that $\partial^{2} \phi / \partial b^{2}=-8 a^{2}-8 a^{4}<0$. Since $\phi(a, 0)=$ $\phi(a, a / 2)=p(a)$ where $p(x)=2 x^{6}-11 x^{4}+12 x^{2}$, we have $\phi \geq p(a)$.

To complete the proof of (9), we will show that $\psi(a, b) \geq d_{0}+1 / 100$. Now

$$
\psi(a, b)=F\left(a, b, b_{m}\right)=a^{2} b_{m}^{2}\left\{4-9 a^{2} / 4-\left(b_{m} / 2+b(b-3 a) /\left(2 b_{m}\right)\right)^{2}\right\}
$$

and calculation shows that $\partial^{2} \psi / \partial b^{2}=-a^{2} b_{m}^{2}+3 a^{2} b^{2}-9 a^{3} b+9 a^{4} / 4$. We shall show that $\partial^{2} \psi / \partial b^{2}<0$, so that $\psi(a, b)$.is a concave function of $b$.

We digress for a moment to show that $b \geq b_{m}(a)$, where $b_{m}=\sqrt{4-b_{m}^{2}}-a$. To see this, recall that for triple packing we must have $b^{2} \geq 4-(a+b)^{2}$, whereas $b^{2} \leq b_{m}^{2}$, since $d(\Lambda) \leq d_{0}$. The juxtaposition $b_{m}^{2} \geq 4-(a+b)^{2}$ yields $b \geq b_{m}$. Putting $b=a / 2$ we see that $b_{m} \leq a / 2$. It is conceivable that $b_{m}$ is negative, even though $b$ never is. For what follows, it is useful to know that $b_{m} \geq-a / 2$. To see this, note that

$$
b_{m}^{2}=a^{2} / 6+(2 / 3) \sqrt{a^{4} / 16+3 d_{0}^{2} / a^{2}}<1 / 6+(2 / 3) \sqrt{1 / 16+27}<3.64,
$$

since $2 / 3 \leq a \leq 1$ and $3<d_{0}^{2}<4$, so that $4-b_{m}^{2}>1 / 4 \geq a^{2} / 4, b_{m}+a=\sqrt{4-b_{m}^{2}}>$ $a / 2$, and $b_{m}>-a / 2$.

We now return to showing that $\psi(a, b)$ is a concave function of $b$ for $b_{m} \leq b$ $\leq a / 2$. Since $\partial^{3} \psi / \partial b^{3}=6 a^{2} b-9 a^{3} \leq 3 a^{3}-9 a^{3}=-6 a^{3}<0$, it is enough to show that $f(a)=\left(1 / a^{2}\right) \partial^{2} \psi /\left.\partial b^{2}\right|_{b=b_{m}}<0$.

Since $f(a)$ is a rather complicated function of the single variable $a$, we shall simply find an upper bound for its derivative as a function of $a$ and use a computer to evaluate it on a fine grid. 
Let $F(x, y, z)=-z+3 y^{2}-9 x y+9 x^{2} / 4$ so that $F\left(a, b_{m}, b_{m}^{2}\right)=f(a)$. Then

$$
\left|f^{\prime}(a)\right| \leq\left|\frac{\partial F}{\partial x}\right|_{0}+\left|\frac{\partial F}{\partial y}\right|_{0}\left|\frac{d b_{m}}{d a}\right|+\left|\frac{\partial F}{\partial z}\right|_{0}\left|\frac{d}{d a} b_{m}^{2}\right|,
$$

where the subscript 0 indicates that the partial derivatives are evaluated at $(x, y, z)=\left(a, b_{m}, b_{m}^{2}\right)$.

Then

$$
\left|\frac{\partial F}{\partial x}\right|_{0}=\left|-9 b_{m}+9 a / 2\right| \leq 9 a \leq 9, \quad\left|\frac{\partial F}{\partial y}\right|_{0} \leq\left|6 b_{m}\right|+9 a \leq 12, \quad \text { and } \quad\left|\frac{\partial F}{\partial z}\right|_{0}=1 \text {. }
$$

Let $u=a^{2}, g(u)=b_{m}^{2}=u / 6+(2 / 3) \sqrt{u^{2} / 16+3 d_{0}^{2} / u}$. Then

$$
g^{\prime}(u)=1 / 6+(1 / 3)\left(u / 8-3 d_{0}^{2} / u^{2}\right) / \sqrt{u^{2} / 16+3 d_{0}^{2} / u},
$$

and

$$
\begin{aligned}
\left|g^{\prime}(u)\right| \leq 1 / 6+ & (1 / 3)\left(1 / 8+3 \times 4 /(4 / 9)^{2}\right) / \sqrt{(4 / 9)^{2} / 16+9} \\
& =1 / 6+(1 / 3)(1 / 8+243 / 4) / \sqrt{9}<7 .
\end{aligned}
$$

Therefore $\left|d g\left(a^{2}\right) / d a\right|=\left|2 a g^{\prime}\left(a^{2}\right)\right|<14$. That is, $\left|d b_{m}^{2} / d a\right|<14$.

Finally, $b_{m}=\sqrt{4-b_{m}^{2}}-a, d b_{m} / d a=-1-(1 / 2)\left(d b_{m}^{2} / d a\right) / \sqrt{4-b_{m}^{2}}$, and $\left|d b_{m} / d a\right| \leq 1+7\left\{4-b_{m}^{2}\right\}^{-1 / 2}<1+7\{0.36\}^{-1 / 2}<13$, since $b_{m}^{2}<3.64$. Hence

$$
\left|f^{\prime}(a)\right| \leq 9+12 \times 13+1 \times 14=179 \text {. }
$$

Using a computer we verified that (allowing for roundoff error) $f\left(a_{i}\right)<-0.2$ at the points $2 / 3=a_{0}<a_{1}<\cdots<a_{n}=1$, where $n=500$, and $\left|a_{i-1}-a_{i}\right|<$ $1 / 1200$ for $1 \leq i \leq n$.

Let $a$ be an arbitrary number in the interval $[2 / 3,1]$. Then $a \in\left[a_{i-1}, a_{i}\right]$ for some $i$, and therefore

$$
f(a)=f\left(a_{i-1}\right)+\int_{a_{i-1}}^{a} f^{\prime}(t) d t \leq-0.2+\frac{179}{1200}<-0.05
$$

Hence $\psi(a, b)$ is a concave function of $b$ as claimed, so $\psi(a, b) \geq$ $\min \left\{\psi\left(a, b_{m}\right), \psi(a, a / 2)\right\}$.

The functions $\psi\left(a, b_{m}\right)$ and $\psi(a, a / 2)$ are also rather complicated functions of the single variable $a$, and they are both above $d_{0}^{2}+1 / 100$. We shall simply find an upper bound for their derivatives as functions of $a^{2}$ or $a$ and use a computer to evaluate them on a fine mesh.

Let $u=a^{2}$ and $f(u)=\psi(a, a / 2)$. Then $f(u)=u g(u) \Omega(u, g(u))-25 u^{3} / 64$ where $g(u)=b_{m}^{2}=u / 6+(2 / 3) \sqrt{u^{2} / 16+3 d_{0}^{2} / u}$, and $\Omega(u, v)=4-(9 / 4) u-v / 4+(5 / 8) u$. Then $f^{\prime}(u)=g(u) \Omega(u, g(u))+u g^{\prime}(u) \Omega(u, g(u))+u g(u)\left\{\partial \Omega / \partial u+g^{\prime}(u) \partial \Omega / \partial v\right\}-75 u^{2} / 64$.

To estimate $\left|f^{\prime}(u)\right|$, we must estimate $g, \Omega$, and their derivatives. We have $|g(u)| \leq 3.64<4$ from before and $|g(u)| \geq u / 3$, trivially. Hence $|\Omega(u, g(u))| \leq$ 
$4+13 u_{\prime}^{\prime} 8+|g(u) / 4| \leq 4+13 / 8+1 \leq 7$. We also have $\left|g^{\prime}(u)\right| \leq 7$ from before. On the other hand $|\partial \Omega / \partial u|=|13 / 8|<2$ and $|\partial \Omega / \partial \nu|=1 / 4$. Putting the estimates together, $\left|f^{\prime}(u)\right|<4 \times 7+7 \times 7+4 \times 2+5+2=92$.

We will show that $f(u) \geq d_{0}^{2}+1 / 100$ for $4 / 9 \leq u \leq 1$. Let us suppose that a computing machine has verified that $f\left(u_{i}\right) \geq d_{0}^{2}+\overline{1} / 50+\epsilon$ for $4 / 9=u_{0}<u_{1}<\ldots<$ $u_{n}=1$, where $\left|u_{i-1}-u_{i}\right|<(50 \times 92)^{-1}$. It then follows that $f(u) \geq d_{0}^{2}+\epsilon$ for $4 / 9$ $\leq u \leq 1$. For $u \in\left[u_{i-1}, u_{i}\right]$ for some $i$, and

$$
\begin{aligned}
& |f(u)| \geq\left|f\left(u_{i-1}\right)\right|-\left|\int_{u_{i-1}}^{u} f^{\prime}(t) d t\right| \\
& \quad \geq d_{0}^{2}+1 / 50+\epsilon-92\left|u_{i-1}-u_{i}\right| \geq d_{0}+\epsilon .
\end{aligned}
$$

A computing machine was programmed to find the minimum value of $f\left(u_{i}\right)$ for $1 \leq i \leq 8251$, where $u_{i}=4 / 9+(i-1) / 14850$, and the answer was 3.51822 .

Had there been no roundoff error, we could say that $f\left(u_{i}\right) \geq d_{0}^{2}+1 / 6$. It is certainly safe to say that $f(u) \geq d_{0}^{2}+1 / 50+1 / 100$. Hence $\psi(a, a / 2)=f\left(a^{2}\right)>$ $d_{0}^{2}+1 / 100$ for $2 / 3 \leq a \leq 1$.

We shall use the same method for $\psi\left(a, b_{m}\right)$. Let us rename $f(a)=\psi\left(a, b_{m}\right)=$ $a^{2} b_{m}^{2}\left\{4-(9 / 4) a^{2}-b_{m}^{2} / 4-b_{m}\left(b_{m}-3 a\right) / 2\right\}-a^{2} b_{m}^{2}\left(b_{m}-3 a\right)^{2} / 4$. It is unfortunate that $\psi\left(a, b_{m}\right)$ cannot be written simply as a function of $a^{2}$; all our functions are now functions of $a$. Let $g_{1}(a)=b_{m}^{2}=g\left(a^{2}\right)$, and let $b(a)=b_{m}$. Put $\Phi(x, y, z)=4$ $-(9 / 4) x^{2}-y / 4-z(z-3 x) / 2$ and $\Theta(x, z)=-x^{2} z^{2}(z-3 x)^{2} / 4$. Then

$$
f(a)=a^{2} g_{1}(a) \Phi\left(a, g_{1}(a), b(a)\right)-\Theta(a, b(a)),
$$

and

$$
\begin{aligned}
f^{\prime}(a)= & 2 a g_{1}(a) \Phi\left(a, g_{1}(a), b(a)\right)+a^{2} g_{1}^{\prime}(a) \Phi\left(a, g_{1}(a), b(a)\right) \\
& +a^{2} g_{1}(a)\left\{\partial \Phi / \partial x+(\partial \Phi / \partial y) g_{1}^{\prime}(a)+(\partial \Phi / \partial z) b^{\prime}(a)\right\}-\partial \Theta / \partial x-(\partial \Theta / \partial z) b^{\prime}(a) .
\end{aligned}
$$

Using some of the estimates from before and making some new ones, we see that $\left|g_{1}(a)\right|=\left|g\left(a^{2}\right)\right|<4,|b(a)|=\left|\sqrt{4-b_{m}^{2}}-a\right| \leq 1 / 2,|\Phi| \leq 4+9 / 4+1+b^{2}(a) / 2+$ $3|b(a)| / 2 \leq 1 / 8+28 / 4<8,\left|g_{1}^{\prime}(a)\right|<14, \partial \Phi / \partial x=9 x / 2+3 z / 2,|\partial \Phi / \partial x| \leq 9 / 2+$ $(3 / 2) \times 1 / 2<6,|\partial \Phi / \partial y|=1 / 4,|\partial \Phi / \partial z|=|-z+3 x / 2| \leq 1 / 2+3 / 2=2$, and $\left|b^{\prime}(a)\right|<13$. By calculation, $\partial \Theta / \partial x=x z^{2}\left(z^{2}-9 x z+18 x^{2}\right) / 2$. Taking the maximum of the positive and negative parts, $|\partial \Theta / \partial x| \leq h_{m}^{2} / 2 \times \max \left\{h_{m}^{2}+18 a^{2}, 9 b_{m}\right\} \leq(1 / 8) \max \{1 / 4+13,9 / 2\}<3$. Similarly, $\partial \Theta / \partial z=-x^{2} z\left(z^{2}-6 z x+9 x^{2}+z^{2}-3 z x\right) / 2$, and $|\partial \Theta / \partial z| \leq\left(b_{m} / 2\right) \max \left\{2 b_{m}^{2}+9,9 b_{m}\right\}<3$. Putting these estimates together, $\left|f^{\prime}(a)\right| \leq 2 \times 4 \times 8+14 \times 13+4(6+14 / 4+2 \times 13)+3+3 \times 13=$ 430. To show that $f(a) \geq d_{0}^{2}+\epsilon$, therefore, it is enough to show that $f\left(a_{i}\right) \geq d_{0}^{2}+1 / 50+\epsilon$ for $2 / 3=a_{0}<\cdots<a_{n}=1$ where $\left|a_{i-1}-a_{i}\right|<1 / 25,000$.

A computing machine was programmed to find the minimum value of $f\left(a_{i}\right)$ for $1 \leq i \leq 100,001$ where $a_{i}=2 / 3+i / 300,000$, and the answer was $3.40344 \ldots$ 
Had there been no roundoff error we could say that $f\left(a_{i}\right) \geq d_{0}^{2}+1 / 15$. It is certainly safe to say that $f\left(a_{i}\right) \geq d_{0}+1 / 50+1 / 100$ so that $\psi\left(a, b_{m}\right)=f(a) \geq d_{0}^{2}+$ $1 / 100$ for $2 / 3 \leq a \leq 1$. Therefore $\psi(a, b) \geq \min \left\{\psi(a, a / 2), \psi\left(a, b_{m}\right)\right\} \geq d_{0}^{2}+1 / 100$ and $f_{2}^{2} \geq \min \{\psi(a, b), \phi(a, b)\} \geq \min \left\{d_{0}^{2}+1 / 100, p(a)\right\}$ as claimed. Thus (9) is proved.

We now prove (10). Let $f(t)=2 t^{3}-11 t^{2}+12 t-d_{0}^{2}$. Then $f^{\prime}(t)=6 t^{2}-22 t+$ 12 and $f^{\prime \prime}(t)=12 t-22<0$ for $0 \leq t \leq 1$. Hence $f(t)$ is a concave function and has at most two zeroes in the range $[0,1]$. In fact, $f(4 / 9)=0$, and $f\left(\alpha^{2}\right)=0$ where $\alpha^{2}=0.90402 \ldots$ and $f(t)>0$ for $4 / 9<t<a^{2}$. Since $f\left(a^{2}\right)=p(a)-d_{0}^{2}$, we conclude that $p(a)>d_{0}^{2}$ for $2 / 3<a<a=0.950802 \ldots$ and (10) is proved.

It now follows from (8), (9) and (10) that $d(\Lambda) \geq d_{0}$ for $2 / 3 \leq a \leq 0.9508$ with equality only when $a=2 / 3$.

\section{REFERENCES}

1. L. Few, Ph. D. Thesis, London, 1953.

2. - Multiple packing of spheres: A survey, Proc. Colloq。 on Convexity (Copenhagen, 1965), Kóbenhavns Univ. Mat. Inst., Copenhagen, 1967, 88-93. MR 35 \#6036.

3. - The double packing of spheres, J. London Math. Soc. 28 (1953), 297-304. MR 14, 1115.

4. L. Few and P. Kanagasabapathy, The double packing of spheres, J. London Math. Soc. 44 (1969), 141-146. MR 39 \#4752.

5. A. Heppes, Mehrfache gitterförmige Kreislargerungen in der Ebene, Acta Math. Acad. Sci. Hungar. 10 (1959), 141-148. MR 21 \#3812。

6. L. E. Dickson, Studies in the theory of numbers, Univ。 of Chicago Press, Chicago, I11., 1939 .

CENTER FOR ADVANCED COMPUTATION, UNIVERSITY OF ILLINOIS AT URBANACHAMPAIGN, URBANA, ILLINOIS 61801 